\title{
INTEGRALS INVOLVING $E$-FUNCTIONS AND MODIFIED BESSEL FUNCTIONS OF THE SECOND KIND
}

by F. M. RAGAB

(Received 4th September, 1953)

§1. Introductory. The two following formulae are to be established.

If $R(m \pm n)>0$, $|\operatorname{amp} z|<\pi$,

$\int_{0}^{\infty} \lambda^{m-1} K_{n}(\lambda) E\left(p ; a_{r}: q ; \rho_{s}: z / \lambda\right) d \lambda$

$=2^{\alpha_{1}+\alpha_{2}+\ldots+\alpha_{p}-\rho_{1}-\ldots-\rho_{q}+q-p+m-2} \pi^{\mathfrak{\natural}(q-p+1)}$

$\left[\begin{array}{c}E\left\{\begin{array}{c}\frac{m+n}{2}, \frac{m-n}{2}, \frac{\alpha_{1}}{2}, \frac{\alpha_{1}+1}{2}, \ldots, \frac{\alpha_{p}}{2}, \frac{\alpha_{p}+1}{2}: e^{ \pm i \pi} \Psi^{q-p} z^{2} \\ \frac{1}{2}, \frac{\rho_{1}}{2}, \frac{\rho_{1}+1}{2}, \ldots, \frac{\rho_{q}}{2}, \frac{\rho_{q}+1}{2}\end{array}\right\} \\ -\frac{2^{p-q}}{z} E\left\{\begin{array}{l}\frac{m+n+1}{2}, \frac{m-n+1}{2}, \frac{\alpha_{1}+1}{2}, \frac{\alpha_{1}+2}{2}, \ldots, \frac{\alpha_{p}+1}{2}, \frac{\alpha_{p}+2}{2}: e^{ \pm i \pi} 4^{q-p_{z}} z^{2} \\ \frac{3}{2}, \frac{\rho_{1}+1}{2}, \frac{\rho_{1}+2}{2}, \ldots, \frac{\rho_{q}+1}{2}, \frac{\rho_{q}+2}{2}\end{array}\right\}\end{array}\right]$

If $p \geqq q+1, R\left(k \pm n+2 \alpha_{r}\right)>0, r=1,2, \ldots, p,|\operatorname{amp} z|<\pi$,

$\int_{0}^{\infty} K_{n}(\lambda) \lambda^{k-1} E\left(p ; \alpha_{r}: q ; \rho_{s}: \lambda^{2} z\right) d \lambda$

$=2^{k-2} \frac{\pi^{2}}{\sin \left(\frac{k+n}{2} \pi\right) \sin \left(\frac{k-n}{2} \pi\right)} E\left(p ; \alpha_{r}: 1-\frac{k+n}{2}, 1-\frac{k-n}{2}, \rho_{1}, \ldots, \rho_{q}: 4 z\right)$

$+\sum_{n,-n} \frac{\pi^{22^{-n-2}} \sin \left(\frac{k+n}{2} \pi\right) \sin (n \pi)}{z^{-(k+n) / 2} E}\left(\begin{array}{c}\alpha_{1}+\frac{k+n}{2}, \ldots, \alpha_{p}+\frac{k+n}{2} \\ 1+\frac{k+n}{2}, n+1, \rho_{1}+\frac{k+n}{2}, \ldots, \rho_{a}+\frac{k+n}{2}: 4 z\end{array}\right)$

For other values of $p$ and $q$ the formula holds if the integral is convergent.

In $\S 2$ these formulae are proved; in $\S 3$ integrals of products of Bessel Functions are evaluated by means of (2).

§2. Proofs. For the first formula, consider the integral

$$
\int_{0}^{\infty} \lambda^{m-1} K_{n}(\lambda) E\left(: q ; \rho_{s}: z / \lambda\right)
$$


where $R(m \pm n)>0$. It can be written

$$
\begin{aligned}
& \int_{0}^{\infty} \lambda^{m-1} K_{n}(\lambda) \frac{1}{\Gamma\left(\rho_{1}\right) \ldots \Gamma\left(\rho_{q}\right)} F\left(; \rho_{1}, \ldots, \rho_{q} ;-\lambda / z\right) d \lambda \\
& =\int_{0}^{\infty} \lambda^{m-1} K_{n}(\lambda) \\
& \times\left[\begin{array}{c}
\frac{1}{\Gamma\left(\rho_{1}\right) \ldots \Gamma\left(\rho_{q}\right)} F\left(; \frac{1}{2}, \frac{\rho_{1}}{2}, \frac{\rho_{1}+1}{2}, \ldots, \frac{\rho_{q}}{2}, \frac{\rho_{q}+1}{2} ; \lambda^{2} z^{-2} 4^{-q-1}\right) \\
-\frac{1}{\Gamma\left(\rho_{1}+1\right) \ldots \Gamma\left(\rho_{q}+1\right)} \frac{\lambda}{z} F\left(; \frac{3}{2}, \frac{\rho_{1}+1}{2}, \frac{\rho_{1}+2}{2}, \ldots, \frac{\rho_{q}+1}{2}, \frac{\rho_{q}+2}{2} ; \lambda^{2} z^{-2} 4^{-q-1}\right)
\end{array}\right] d \lambda .
\end{aligned}
$$

On expanding term by term and applying the formula (1)

$$
\int_{0}^{\infty} \lambda^{m-1} K_{n}(\lambda) d \lambda=2^{m-2} \Gamma\left(\frac{m+n}{2}\right) \Gamma\left(\frac{m-n}{2}\right)
$$

where $R(m \pm n)>0$, the value of the integral is found to be

$$
\begin{aligned}
& 2^{m}{ }^{2} \Gamma\left(\frac{m+n}{2}\right) \Gamma\left(\frac{m-n}{2}\right) \frac{\pi^{\frac{1}{2}}}{\Gamma\left(\frac{1}{2}\right) \Gamma\left(\rho_{1}\right) \ldots \Gamma\left(\rho_{q}\right)} F\left(\begin{array}{l}
12 m+\frac{1}{2} n, \frac{1}{2} m-\frac{1}{2} n ; \frac{1}{z^{2} 4^{q}} \\
\frac{1}{2}, \frac{\rho_{1}}{2}, \ldots, \frac{\rho_{q}+1}{2}
\end{array}\right) \\
& 2^{m-1} \Gamma\left(\frac{m+n+1}{2}\right) \Gamma\left(\frac{m-n+1}{2}\right) \frac{\pi^{\frac{3}{2}}}{\Gamma\left(\frac{3}{2}\right) \Gamma\left(\rho_{1}+1\right) \ldots \Gamma\left(\rho_{q}+1\right)}\left(\frac{1}{2 z}\right) \\
& \times F\left(\begin{array}{l}
\frac{1}{2} m+\frac{1}{2} n+\frac{1}{2}, \frac{1}{2} m-\frac{1}{2} n+\frac{1}{2} ; \frac{1}{z^{2} 4^{q}} \\
\frac{3}{2}, \frac{\rho_{1}+1}{2}, \ldots, \frac{\rho_{q}+2}{2}
\end{array}\right) \\
& =2^{m-2-\rho_{1}-\ldots-p_{q}+q_{\pi^{\mathrm{b}}(q+1)}}\left\{\begin{array}{l}
E\left(\frac{m+n}{2}, \frac{m-n}{2} ; \frac{1}{2}, \frac{\rho_{1}}{2}, \ldots, \frac{\rho_{q}+1}{2}: e^{ \pm i \pi_{4} q^{2}}\right) \\
-\frac{2^{-q}}{z} E\left(\frac{m+n+1}{2}, \frac{m-n+1}{2}: \frac{3}{2}, \frac{\rho_{1}+1}{2}, \ldots, \frac{\rho_{q}+2}{2}: e^{ \pm i \pi} 4^{q_{\kappa}} z^{2}\right.
\end{array}\right\} .
\end{aligned}
$$

On making repeated applications of the formula, (2),

$$
\begin{aligned}
\int_{0}^{\infty} e^{-\lambda} \lambda^{k-1} E & \left(p ; \alpha_{r}: q ; \rho_{s}: z / \lambda^{m}\right) d \lambda \\
= & m^{k-\frac{1}{1}}(2 \pi)^{\frac{1-1}{2} m} E\left(p+m ; \alpha_{r}: q ; \rho_{s}: z / m^{m}\right),
\end{aligned}
$$

where $m$ is a positive integer ( 1 and 2 in this case), $R(k)>0, \alpha_{p+v+1}=(k+v) / m, v=0,1, \ldots, m-1$, formula (1) is obtained.

Note. The method can be employed to express the integral as the sum of any number of $E$-functions.

In the proof of (2) the following formulae are required.

(2) If $m$ is a positive integer and if $R\left(k_{ \pm n)}>0\right.$,

$$
\begin{aligned}
\int_{0}^{\infty} K_{n}(\lambda) \lambda^{k-1} E(p & \left.; \alpha_{r}: q ; \rho_{s}: z / \lambda^{2 m}\right) d \lambda \\
& =(2 \pi)^{1-m} 2^{k-2} m^{k-1} E\left\{p+2 m ; \alpha_{r}: q ; \rho_{s}: z /(2 m)^{2 m}\right\},
\end{aligned}
$$

where $\alpha_{p+v+1}=(k+n+2 v) /(2 m), \quad \alpha_{p+m+v+1}=(k-n+2 v) /(2 m), v=0,1,2, \ldots, m-1$. 
(3) If $p \geqq q+1$,

$$
\begin{aligned}
E\left(p ; \alpha_{r}: q ; \rho_{s}: z\right)=\sum_{r=1}^{p} \prod_{s=1}^{p} \Gamma\left(\alpha_{s}-\alpha_{r}\right)\left\{\prod_{t=1}^{q} \Gamma\left(\rho_{t}-\alpha_{r}\right)\right\}^{-1} \Gamma\left(\alpha_{r}\right) \\
\quad \times z^{\alpha_{r} F}\left\{\begin{array}{l}
q+1 ; \alpha_{r}, \alpha_{r}-\rho_{1}+1, \ldots, \alpha_{r}-\rho_{q}+1:(-1)^{p-q_{z}} \\
p-1 ; \alpha_{r}-\alpha_{1}+1, \ldots * \ldots, \alpha_{r}-\alpha_{p}+1
\end{array}\right\},
\end{aligned}
$$

and if $p \leqq q$,

$$
E\left(p ; \alpha_{r}: q ; \rho_{s}: z\right)=\frac{\Gamma\left(\alpha_{1}\right) \ldots \Gamma\left(\alpha_{p}\right)}{\Gamma\left(\rho_{1}\right) \ldots \Gamma\left(\rho_{q}\right)} F\left(p ; \alpha_{r}: q: \rho_{s}:-\frac{1}{z}\right) .
$$

When $p=1, q=0$, the integral in (2) becomes

$$
\begin{aligned}
& \int_{0}^{\infty} K_{n}(\lambda) \lambda^{k-1} E\left(\alpha_{1}:: \lambda^{2} z\right) d \lambda \\
& =z^{\alpha_{1}} \int_{0}^{\infty} K_{n}(\lambda) \lambda^{k+2 \alpha_{1}-1} E\left\{\alpha_{1}:: 1 /\left(z \lambda^{2}\right)\right\} d \lambda
\end{aligned}
$$

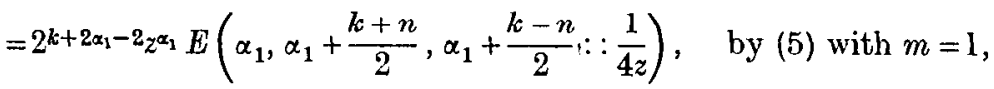

$$
\begin{aligned}
& =2^{k-2} \dot{\Gamma}\left(\frac{k+n}{2}\right) \Gamma\left(\frac{k-n}{2}\right) \Gamma\left(\alpha_{1}\right) F\left(\alpha_{1} ; 1-\frac{k+n}{2}, 1-\frac{k-n}{2} ;-\frac{1}{4 z}\right) \\
& +\sum_{n,-n} 2^{-n-2} \Gamma\left(\frac{-k-n}{2}\right) \Gamma(-n) \Gamma\left(\alpha_{1}+\frac{k+n}{2}\right) z^{-\frac{1}{\mathbf{b}-b^{n}} F}\left(\alpha_{1}+\frac{k+n}{2} ; 1+\frac{k+n}{2}, n+1 ;-\frac{1}{4 z}\right) \text {, } \\
& =2^{k-2} \frac{\pi^{2}}{\sin \left(\frac{k+n}{2} \pi\right) \sin \left(\frac{k-n}{2} \pi\right)} E\left(\alpha_{1}: 1-\frac{k+n}{2}, 1-\frac{k-n}{2}: 4 z\right) \\
& +\sum_{n,-n} \frac{\pi^{2} 2^{-n-2}}{\sin \left(\frac{k+n}{2} \pi\right) \sin (n \pi)} z^{-\frac{k}{k}-k^{n}} E\left(\alpha_{1}+\frac{k+n}{2}: 1+\frac{k+n}{2}, n+1: 4 z\right), \quad \text { by }(6 \mathrm{~b}) \text {. }
\end{aligned}
$$

From this (2) can be derived in the usual way.

$\S 3$. Some Bessel Function Integrals. In (2) take $p=0, q=1$, replace $z$ by $4 / z^{2}, n$ by $m$ and put $\rho_{1}=n+1$; then, on applying the formula

$$
E\left(: n+1: 4 \lambda^{2} / z^{2}\right)=(2 \lambda / z)^{n} J_{n}(z / \lambda),
$$

it is found that, if $z$ is real and positive, $R(k+n \pm m)>-\frac{3}{2}$,

$$
\begin{aligned}
(2 / z)^{n} \int_{0}^{\infty} K_{m}(\lambda) \lambda^{k+n-1} J_{n}(z / \lambda) d \lambda & \\
= & \frac{2^{k-2} \pi^{2}}{\sin \left(\frac{k+m}{2} \pi\right) \sin \left(\frac{k-m}{2} \pi\right)} E\left(: 1-\frac{k+m}{2}, 1-\frac{k-m}{2}, n+1: 16 / z^{2}\right) \\
& \quad+\sum_{m,-m} \frac{2^{-m-2} \pi^{2}}{\sin \left(\frac{k+m}{2} \pi\right) \sin (m \pi)}\left(\frac{z}{2}\right)^{k+m} E\left(: 1+\frac{k+m}{2}, m+1,1+n+\frac{k+m}{2}: 16 / z^{2}\right) .
\end{aligned}
$$


Here replace $k$ by $k-n$; then, if $z$ is real and positive, $R(k \pm m)>-\frac{3}{2}$,

$$
\begin{aligned}
\int_{0}^{\infty} & \lambda^{k-1} K_{m}(\lambda) J_{n}(z / \lambda) d \lambda \\
= & \frac{\Gamma\left(\frac{k+m-n}{2}\right) \Gamma\left(\frac{k-m-n}{2}\right)}{2^{2 n-k+2} \Gamma(n+1)} z^{n} F\left(; 1-\frac{k+m-n}{2}, 1-\frac{k-m-n}{2}, n+1 ;-\frac{z^{2}}{16}\right) \\
& +\sum_{m,-m} \frac{\Gamma\left(\frac{-k-m+n}{2}\right) \Gamma(-m)}{\Gamma\left(1+\frac{k+m+n}{2}\right)} \frac{z^{k+m}}{2^{k+2 m+2}} F\left(; 1+\frac{k+m-n}{2}, 1+\frac{k+m+n}{2}, m+1 ;-\frac{z^{2}}{16}\right) .
\end{aligned}
$$

On applying the formula

$$
G_{n}(z)=\frac{\pi}{2 \sin n \pi}\left\{J_{-n}(z)-e^{-i n \pi} J_{n}(z)\right\},
$$

it follows that, if $0 \leqq \operatorname{amp} z \leqq \pi, R(k \pm m)>-\frac{3}{2}$,

$$
\begin{aligned}
& i^{n} \int_{0}^{\infty} \lambda^{k-1} K_{m}(\lambda) G_{n}(z / \lambda) d \lambda \\
&=\sum_{n,-n} 2^{k+2 n-3} \Gamma\left(\frac{k+m+n}{2}\right) \Gamma\left(\frac{k-m+n}{2}\right) \Gamma(n)\left(\frac{i}{z}\right)^{n} \\
& \times F\left(; 1-\frac{k+m+n}{2}, 1-\frac{k-m+n}{2}, 1-n ;-\frac{z^{2}}{16}\right) \\
&+\sum_{m,-m} \frac{\pi}{2 \sin n \pi} \Gamma\left(\frac{-k-m-n}{2}\right) \Gamma\left(\frac{-k-m+n}{2}\right) \Gamma(-m) \frac{z^{k+m}}{2^{k+2 m+2}} \\
& \times F\left(; 1+\frac{k+m+n}{2}, 1+\frac{k+m-n}{2}, m+1 ;-\frac{z^{2}}{16}\right) \\
& \times \frac{1}{\pi}\left[-\sin \left(\frac{k+m-n}{2} \pi\right) e^{i n \pi / 2}+\sin \left(\frac{k+m+n}{2} \pi\right) e^{-i n \pi / 2}\right] .
\end{aligned}
$$

Now the expression in the bracket is equal to

$$
\sin n \pi i^{-k-m} \text {. }
$$

Hence, on replacing $z$ by $i z$, and noting that

the formula becomes (4)

$$
G_{n}(i z)=i^{-n} K_{n}(z)
$$

$$
\begin{aligned}
& \int_{0}^{\infty} \lambda^{k-1} K_{m}(\lambda) K_{n}(z / \lambda) d \lambda \\
& =\sum_{n,-n} 2^{k+2 n-3} \Gamma\left(\frac{k+m+n}{2}\right) \\
& \times\left(\frac{k-m+n}{2}\right) \Gamma(n) z^{-n} \\
& \times F\left(; 1-\frac{k+m+n}{2}, 1-\frac{k-m+n}{2}, 1-n ; \frac{z^{2}}{16}\right) \\
& +\sum_{m,-m} 2^{-k-2 m-3} \Gamma\left(\frac{-k-m-n}{2}\right) \Gamma\left(\frac{-k-m+n}{2}\right) \Gamma(-m) z^{k+m} \\
& \times F\left(; 1+\frac{k+m+n}{2}, 1+\frac{k+m-n}{2}, m+1 ; \frac{z^{2}}{16}\right),
\end{aligned}
$$

where $R(z)>0$. 


\section{REFERENCES}

(1) Gray, Mathews and MacRobert, Bessel Functions, p. 66.

(2) MacRobert, T. M., Proc. Glasg. Math. Ass., 1, 191 (1953).

(3) MacRobert, T. M., Functions of a Complex Variable (3rd ed., London, 1946), formulae (21), (22), (23), pp. 352, 353 .

(4) MacRobert, T. M., Proc. Glasg. Math. Ass., 1, 187 (1953).

\section{UNIVERSITY OF GLaSgow \\ and \\ Faculty of Science \\ IBRAHIM UNIVERSITY \\ CAIro}

